OCU-PHYS-236 , AP-GR-28， YITP-06-03

\title{
Naked singularity resolution in cylindrical collapse
}

\author{
Yasunari Kurita ${ }^{1,2 *}$ and Ken-ichi Nakao $1+$ \\ ${ }^{1}$ Department of Physics, Osaka City University, Osaka 558-8585, Japan \\ ${ }^{2}$ Yukawa Institute for Theoretical Physics, \\ Kyoto University, Kyoto, 606-8502, Japan
}

\begin{abstract}
In this paper, we study the gravitational collapse of null dust in the cylindrically symmetric spacetime. The naked singularity necessarily forms at the symmetry axis. We consider the situation in which null dust is emitted again from the naked singularity formed by the collapsed null dust and investigate the back-reaction by this emission for the naked singularity. We show a very peculiar but physically important case in which the same amount of null dust as that of the collapsed one is emitted from the naked singularity as soon as the ingoing null dust hits the symmetry axis and forms the naked singularity. In this case, although this naked singularity satisfies the strong curvature condition by Królak (limiting focusing condition), geodesics which hit the singularity can be extended uniquely across the singularity. Therefore we may say that the collapsing null dust passes through the singularity formed by itself and then leaves for infinity. Finally the singularity completely disappears and the flat spacetime remains.
\end{abstract}

PACS numbers: 04.20.Dw

${ }^{*}$ Electronic address: kurita at’sci.osaka-cu.ac.jp

${ }^{\dagger}$ Electronic address: knakao at sci.osaka-cu.ac.jp 


\section{INTRODUCTION}

It is important to investigate the gravitational collapse in general relativity. One of the most important motivations is to examine the cosmic censorship hypothesis [1]. However, previous theoretical studies have implied the existence of counter examples to the hypothesis [2]-[5]. If the universe is not censored, we can not deny the possibility that a spacetime singularity is observable. The observable singularity is called the naked singularity. By its definition, there are regions which suffer the physical effects of naked singularities. Therefore, in order to construct a solution describing a spacetime accompanied by naked singularities by solving Einstein equations as a Cauchy problem, we need to specify the boundary condition at the naked singularity. In other words, in order to predict the dynamics of the spacetime accompanied by the naked singularity, we need the knowledge about the spacetime singularity. However all known theories of physics including general relativity are not applicable at the spacetime singularity. This fact is not a serious problem. Conversely the appearance of naked singularities might give us important information about new physics

6]. The knowledge of the naked singularity is very important even within the framework of general relativity.

It is well known that, in the case of the cylindrical gravitational collapse, a resultant singularity is always naked [7, 8]. In the literature, there are a few exact solutions for cylindrical gravitational collapse. One of them gives a description of a cylindrical null dust collapse, originally constructed by Morgan [9]. The feature of the singularity formed by the gravitational collapse of this null dust were studied by Letelier and Wang[10]. They showed that in the case with only ingoing null dust, the singularity is the p.p. curvature singularity which is defined by the behavior of components of the Riemann tensor with respect to parallelly propagated tetrad frame, while in the case of the coexistence of ingoing and outgoing null dust, the singularity is the scalar polynomial (s.p.) curvature singularity which is defined by the behavior of the scalar polynomials constructed by the Riemann tensor[11]. The global structure of the spacetime with only ingoing null dust has been studied by Nolan[14].

In this paper, we study the feature of the Riemann tensor and geodesics near the singularity in Morgan's spacetime from different point of view of Letelier and Wang. The assumption of only ingoing null dust corresponds to the boundary condition in which all the null dust 
is absorbed by the singularity formed by itself. However, of course, this boundary condition is not necessarily unique since, as mentioned, we do not know how to impose the boundary condition at the naked singularity. Keeping this fact in mind, there is a possibility that something is emitted from the naked singularity formed by the collapsed null dust although we do not know what something is at present. In this paper, we assume that the emitted matter or radiation is also null dust and study how the feature of the naked singularity changes by the amount of the emitted null dust.

The paper is organized as follows. Section पis devoted to a review of the cylindrical null dust collapse. In this section, in order to see the curvature strength of the naked singularity, we also investigate the components of the Riemann tensor. In section IIII, we investigate radial geodesics and their extendibility across the singularity. Section [V summarizes the paper. Appendix A gives a description of geodesics in the case of the Morgan's spacetime, which is used in section II. In Appendix B, we show the behavior of non-radial geodesics near the singularity. In Appendix C, we show the convergence of the Weyl tensor along null geodesics hitting the naked singularity.

In this paper, we adopt $c=1$ unit and follow the convention of the Riemann and metric tensors in Ref. 11]. The Greek indices mean general coordinate basis components while the Latin indices will be used to denote specific coordinate basis components.

\section{NAKED SINGULARITY IN CYLINDRICAL NULL DUST COLLAPSE}

Let us consider the spacetime with whole cylinder symmetry [12, 13] and further, as mentioned, we assume the null dust. Then the line element of this spacetime is given as

$$
d s^{2}=e^{2 \gamma}\left(-d t^{2}+d r^{2}\right)+r^{2} d \phi^{2}+d z^{2}
$$

where $\gamma$ is a function of $t$ and $r$. The energy-momentum tensor of null dust is

$$
T_{\mu \nu}=\frac{1}{\kappa r}\left(A k_{\mu} k_{\nu}+B \ell_{\mu} \ell_{\nu}\right)
$$

where $\kappa$ is the gravitational constant and $k_{\mu}$ is an ingoing null vector, $k_{\mu}=(-1,-1,0,0)$, while $\ell_{\mu}$ is an outgoing null vector, $\ell_{\mu}=(-1,1,0,0)$. The strong, weak and dominant energy conditions are all equivalent to $A \geq 0$ and $B \geq 0$. In the following, we assume these conditions. 
Hereafter we shall also use null coordinates,

$$
\begin{aligned}
& u=t-r, \\
& v=t+r .
\end{aligned}
$$

The local conservation law $T_{\mu}{ }^{\nu}{ }_{; \nu}=0$ leads $\left(\partial_{u} A\right)_{v}=0$ and $\left(\partial_{v} B\right)_{u}=0$, and thus, $A=A(v)$ and $B=B(u)$. We assume appropriate differentiability more than $C^{1}$ for $A(x)$ and $B(x)$ in $-\infty<x<+\infty$. In this case, the Einstein equations demand that $A$ and $B$ are expressed in terms of the metric function $\gamma$ as

$$
A=\left(\partial_{v} \gamma\right)_{u}, \quad B=-\left(\partial_{u} \gamma\right)_{v}
$$

The above equations and the local conservation law demand $\partial_{u} \partial_{v} \gamma=0$ which is consistent with the Einstein equations. The above equations also demand that $\gamma(x, y)$ is at least $C^{2}$ function defined in $-\infty<x<+\infty$ and $-\infty<y<+\infty$. The solution for $\gamma$ is then written as

$$
\gamma=\alpha(v)+\beta(u)
$$

and therefore we obtain

$$
A(v)=\alpha_{, v}, \quad B(u)=-\beta_{, u},
$$

where a comma means the ordinary derivative with respect to the variable following it.

It is seen from eq.(2) that if $A$ or $B$ do not vanish at $r=0$, the coordinate basis components of the energy-momentum tensor diverge at $r=0$. This implies that the spacetime singularity appears at the symmetry axis $r=0$. Indeed, Leterier and Wang showed that the curvature singularity appears there [10]. As mentioned in the previous section, the singularity is p.p. curvature one in the case with only one of the ingoing null dust and outgoing one, while the singularity is s.p. curvature singularity if the ingoing null dust coexists with the outgoing one at the symmetry axis. This singularity is, of course, naked, i.e., in principle, observable.

In this paper, we assume that the null dust takes the form of a cylindrical shell with finite thickness. This is equivalent to the assumption of the compact supports of $A$ and $B$. Further we assume the situation in which before the ingoing null dust collapses to form the singularity at the symmetry axis $r=0$, there is no spacetime singularity. Morgan showed that a conical singularity forms at the symmetry axis after all of the null dust collapses, in 
the case of no outgoing null dust, i.e., $B=0[9]$. However, as mentioned in the previous section, the law of physics at the spacetime singularity has not yet been known. Something might be emitted from the naked singularity and here we assume that this something is also the null dust and investigate the back-reaction effect on the naked singularity by the emission of the null dust.

We investigate the curvature strength of the naked singularity. For this purpose, we consider an observer freely falling radially to the naked singularity, where 'radially' means vanishing $\phi$-component of the 4 -velocity vector of the observer. The coordinate basis components of the 4-velocity vector of this observer are given by

$$
\begin{aligned}
\frac{d t}{d \tau} & =\frac{\left(1+V_{(\mathrm{r})}\right) e^{-2 \alpha}+\left(1-V_{(\mathrm{r})}\right) e^{-2 \beta}}{2 \sqrt{1-V^{2}}}, \\
\frac{d r}{d \tau} & =\frac{\left(1+V_{(\mathrm{r})}\right) e^{-2 \alpha}-\left(1-V_{(\mathrm{r})}\right) e^{-2 \beta}}{2 \sqrt{1-V^{2}}}, \\
\frac{d z}{d \tau} & =\frac{V_{(\mathrm{z})}}{\sqrt{1-V^{2}}},
\end{aligned}
$$

where $\tau$ is the proper time of the observer, and $V_{(\mathrm{r})}$ and $V_{(\mathrm{z})}$ are arbitrary constants, and $V^{2}=$ $V_{(\mathrm{r})}^{2}+V_{(\mathrm{z})}^{2}$. Note that $V^{2}$ should be smaller than unity. The derivation of the above solutions is given in Appendix A. Then we find the behavior of the Ricci tensor in approaching to the naked singularity as

$$
\begin{aligned}
\lim _{\tau \rightarrow 0} \tau R_{\mu \nu} \frac{d x^{\mu}}{d \tau} \frac{d x^{\nu}}{d \tau} & =\lim _{\tau \rightarrow 0} \frac{\tau}{r} \cdot \frac{\left(1+V_{(\mathrm{r})}\right)^{2} \alpha_{, v} e^{-4 \alpha}-\left(1-V_{(\mathrm{r})}\right)^{2} \beta_{, u} e^{-4 \beta}}{1-V^{2}} \\
& =\left[\lim _{\tau \rightarrow 0} \frac{d \tau}{d r}\right]\left[\lim _{\tau \rightarrow 0} \frac{\left(1+V_{(\mathrm{r})}\right)^{2} A e^{-4 \alpha}+\left(1-V_{(\mathrm{r})}\right)^{2} B e^{-4 \beta}}{1-V^{2}}\right] \\
& =\left.\frac{2}{\sqrt{1-V^{2}}} \cdot \frac{\left(1+V_{(\mathrm{r})}\right)^{2} A e^{-4 \alpha}+\left(1-V_{(\mathrm{r})}\right)^{2} B e^{-4 \beta}}{\left(1+V_{(\mathrm{r})}\right) e^{-2 \alpha}-\left(1-V_{(\mathrm{r})}\right) e^{-2 \beta}}\right|_{r=0},
\end{aligned}
$$

where the proper time $\tau$ is adjusted as it equals zero at $r=0$. Equation (17) and the l'Hospital theorem has been used in the second line. The final line also shows that the above limit does not vanish in the region filled with the null dust since $A \geq 0$ and $B \geq 0$. This is a sufficient condition for the strong curvature condition by Królak, which is often called the limiting focusing condition (LFC) [15, 16]. In this sense, this naked singularity is strong.

However, this singularity satisfies neither the strong limiting forcusing condition (SLFC) nor the strong curvature condition by Tipler (SCST). We say that SLFC is satified if the following integral defined on a future directed null geodesic terminating at the singularity is 
unbounded:

$$
\lim _{\lambda \rightarrow 0} \int_{\lambda_{0}}^{\lambda} d \lambda^{\prime} \int_{\lambda_{0}}^{\lambda^{\prime}} d \lambda^{\prime \prime} R_{\mu \nu} \frac{d x^{\mu}}{d \tau} \frac{d x^{\nu}}{d \tau}
$$

where the affine parameter $\lambda$ is adjusted as it equals zero at $r=0$ and $\lambda_{0}$ is some negative value. The components of the tangent vector for the radial null geodesic are

$$
\begin{aligned}
& \frac{d t}{d \lambda}=\frac{1}{2}\left[\left(\omega+k_{(\mathrm{r})}\right) e^{-2 \alpha(v)}+\left(\omega-k_{(\mathrm{r})}\right) e^{-2 \beta(u)}\right] \\
& \frac{d r}{d \lambda}=\frac{1}{2}\left[\left(\omega+k_{(\mathrm{r})}\right) e^{-2 \alpha(v)}-\left(\omega-k_{(\mathrm{r})}\right) e^{-2 \beta(u)}\right] \\
& \frac{d z}{d \lambda}=k_{(\mathrm{z})},
\end{aligned}
$$

where $\omega, k_{(\mathrm{r})}$ and $k_{(\mathrm{z})}$ are integration constants which satisfy $\omega^{2}=k_{(\mathrm{r})}^{2}+k_{(\mathrm{z})}^{2}$. These solutions are derived in Appendix A. Then, the Ricci tensor behaves along the null geodesic as

$$
\lim _{\lambda \rightarrow 0} \lambda R_{\mu \nu} \frac{d x^{\mu}}{d \lambda} \frac{d x^{\nu}}{d \lambda}=\left.2 \cdot \frac{\left(\omega+k_{(r)}\right)^{2} e^{-4 \alpha} A+\left(\omega-k_{(r)}\right)^{2} e^{-4 \beta} B}{\left(\omega+k_{(r)}\right) e^{-2 \alpha}-\left(\omega-k_{(r)}\right) e^{-2 \beta}}\right|_{r=0}=: X .
$$

Thus, the above limit does not vanish as in the case of timelike geodesics. The limiting value is named $X$. In order to show that the singularity does not satisfy the SLFC, let us consider the following limit:

$$
\lim _{\lambda \rightarrow 0} \frac{\int_{\lambda_{0}}^{\lambda} d \lambda^{\prime} \int_{\lambda_{0}}^{\lambda^{\prime}} d \lambda^{\prime \prime} R_{\mu \nu} \frac{d x^{\mu}}{d \lambda} \frac{d x^{\nu}}{d \lambda}}{\lambda \ln \lambda-\lambda}=\lim _{\lambda \rightarrow 0} \frac{\int_{\lambda_{0}}^{\lambda} d \lambda^{\prime \prime} R_{\mu \nu} \frac{d x^{\mu}}{d \lambda} \frac{d x^{\nu}}{d \lambda}}{\ln \lambda}=X,
$$

where the l'Hospital theorem has been used. Therefore,

$$
\lim _{\lambda \rightarrow 0} \int_{\lambda_{0}}^{\lambda} d \lambda^{\prime} \int_{\lambda_{0}}^{\lambda^{\prime}} d \lambda^{\prime \prime} R_{\mu \nu} \frac{d x^{\mu}}{d \lambda} \frac{d x^{\nu}}{d \lambda}=\lim _{\lambda \rightarrow 0}(X(\lambda \ln \lambda-\lambda))=0 .
$$

Thus, the integral (12) is integrable and the strong limiting forcusing condition is not satisfied. Additionally, the integral for the Weyl tensors

$$
L_{b}^{a}=\lim _{\lambda \rightarrow 0} \int_{\lambda_{0}}^{\lambda} d \lambda^{\prime} \int_{\lambda_{0}}^{\lambda^{\prime}} d \lambda^{\prime \prime}\left(\int_{\lambda_{0}}^{\lambda^{\prime \prime}} d \lambda^{\prime \prime \prime}\left|C_{\mu b \nu}^{a} \frac{d x^{\mu}}{d \lambda} \frac{d x^{\nu}}{d \lambda}\right|\right)^{2}
$$

converges for all $a$ and $b$ as shown in Appendix C. And now, a necessary condition for SCST is that either the integral (12) or (19) does not converge [16]. Therefore, this singularity does not satisfy the necessary condition for SCST.

In the above argument, we discuss only for the null geodesic. As we show in Appendix D, the singularity also does not satisfy the necessary condition in the case of timelike geodesic. Thus, the singularity does not satisfy SCST. 


\section{GEODESICS IN THE NEIGHBORHOOD OF THE SINGULARITY}

Here we focus on the radial null and timelike geodesics which necessarily hit the singularity at $r=0$. In order to see the behavior of those near the singularity, let us adopt a Cartesian coordinate instead of the cylindrical coordinate (11), which is defined by

$$
x:=r \cos \phi, \quad y:=r \sin \phi .
$$

Then, the components of the metric tensor with respect to the new coordinate basis are given as

$$
\begin{aligned}
& g_{x x}=\frac{x^{2} e^{2 \gamma}+y^{2}}{r^{2}}=e^{2 \gamma} \cos ^{2} \phi+\sin ^{2} \phi \\
& g_{x y}=\frac{x y\left(e^{2 \gamma}-1\right)}{r^{2}}=\left(e^{2 \gamma}-1\right) \sin \phi \cos \phi \\
& g_{y y}=\frac{x^{2}+e^{2 \gamma} y^{2}}{r^{2}}=\cos ^{2} \phi+e^{2 \gamma} \sin ^{2} \phi .
\end{aligned}
$$

The Christoffel symbols in this coordinate system are given as follows:

$$
\begin{aligned}
& \Gamma_{t t}^{t}=\dot{\gamma}, \quad \Gamma_{t x}^{t}=\gamma^{\prime} \cos \phi, \quad \Gamma_{t y}^{t}=\gamma^{\prime} \sin \phi \\
& \Gamma_{x x}^{t}=\dot{\gamma} \cos ^{2} \phi, \quad \Gamma_{x y}^{t}=\dot{\gamma} \sin \phi \cos \phi, \quad \Gamma_{y y}^{t}=\dot{\gamma} \sin ^{2} \phi \\
& \Gamma_{t t}^{x}=\gamma^{\prime} \cos \phi, \quad \Gamma_{t x}^{x}=\dot{\gamma} \cos ^{2} \phi, \quad \Gamma_{t y}^{x}=\dot{\gamma} \sin \phi \cos \phi \\
& \Gamma_{x x}^{x}=\cos \phi\left(\gamma^{\prime} \cos ^{2} \phi+\frac{1-e^{-2 \gamma}}{r} \sin ^{2} \phi\right), \\
& \Gamma_{x y}^{x}=\sin \phi \cos ^{2} \phi\left(\gamma^{\prime}-\frac{1-e^{-2 \gamma}}{r}\right), \\
& \Gamma_{y y}^{x}=\cos \phi\left(\gamma^{\prime} \sin ^{2} \phi+\frac{1-e^{-2 \gamma}}{r} \cos ^{2} \phi\right), \\
& \Gamma_{t t}^{y}=\gamma^{\prime} \sin \phi, \quad \Gamma_{t x}^{y}=\dot{\gamma} \sin \phi \cos \phi, \quad \Gamma_{t y}^{y}=\dot{\gamma} \sin ^{2} \phi \\
& \Gamma_{x x}^{y}=\sin \phi\left(\gamma^{\prime} \cos ^{2} \phi+\frac{1-e^{-2 \gamma}}{r} \sin ^{2} \phi\right), \\
& \Gamma_{x y}^{y}=\sin \phi \cos \phi\left(\gamma^{\prime}-\frac{1-e^{-2 \gamma}}{r}\right), \\
& \Gamma_{y y}^{y}=\sin \phi\left(\gamma^{\prime} \sin ^{2} \phi+\frac{1-e^{-2 \gamma}}{r} \cos ^{2} \phi\right),
\end{aligned}
$$

and the other components vanish, where $\dot{\gamma}=\left(\partial_{t} \gamma\right)_{r}$ and $\gamma^{\prime}=\left(\partial_{r} \gamma\right)_{t}$.

By virtue of the cylindrical symmetry, it is sufficient for our purpose to consider only the case in which the initial data is given by $x<0, y=0, d x / d \tau>0$ and $d y / d \tau=0$ at 
some proper time $\tau$. We can easily see that $y$ remains zero over the singularity $r=0$ since $\Gamma_{\mu \nu}^{y}=0$ on $y=0$. Due to the translational symmetry of $z$-direction, $d z / d \tau$ is constant and is denoted by the constant $V_{(\mathrm{z})}$ as in the previous section. Then the remaining geodesic equations are given by

$$
\begin{aligned}
& \frac{d^{2} t}{d \tau^{2}}=-\dot{\gamma}\left(\frac{d t}{d \tau}\right)^{2}-2 \frac{x}{|x|} \gamma^{\prime} \frac{d t}{d \tau} \frac{d x}{d \tau}-\dot{\gamma}\left(\frac{d x}{d \tau}\right)^{2} \\
& \frac{d^{2} x}{d \tau^{2}}=-\frac{x}{|x|} \gamma^{\prime}\left(\frac{d t}{d \tau}\right)^{2}-2 \dot{\gamma} \frac{d t}{d \tau} \frac{d x}{d \tau}-\frac{x}{|x|} \gamma^{\prime}\left(\frac{d x}{d \tau}\right)^{2}
\end{aligned}
$$

The right hand sides of the above equations are multi-valued at $x=0$ due to $x /|x|$. However those are finite and thus the solutions for $d t / d \tau$ and $d x / d \tau$ are $C^{1-}$ functions of $\tau$. This result seems to be likely that the geodesics which hit the singularity $r=0$ are uniquely extendible across the singularity, but it is no true except for the case of $\left.\gamma\right|_{r=0}=0$.

In order to see this fact, we explicitly write down the norm $N$ of the geodesic tangent at $r=0$ as

$$
N=\left.\left[-e^{2 \gamma}\left(\frac{d t}{d \tau}\right)^{2}+\left(e^{2 \gamma} \cos ^{2} \phi+\sin ^{2} \phi\right)\left(\frac{d x}{d \tau}\right)^{2}\right]\right|_{r=0}+V_{(\mathrm{z})}^{2},
$$

where we should note that arbitrary values of $\phi$ are assigned for a point on the symmetry axis $r=0$ and thus $\phi$ appears in the right hand side of the above equation. Since $d t / d \tau$ and $d x / d \tau$ are continuous, it is seen from eq.(36) that the norm $N$ becomes multi-valued at $r=0$ as long as $\gamma$ does not vanish at the symmetry axis $r=0$. Here it should be noted that the norm of the affinely parametrized geodesic tangent is preserved. Therefore geodesics which hit the naked singularity at $r=0$ with non-vanishing $\gamma$ can not be extended across this naked singularity since the statement that the geodesic tangent is parallelly propagated is meaningless there. Expressed in another way, the spacetime is conical around $r=0$. The reason of the incompleteness is the same as that of the fact that any spacetime with conical singularities is geodesically incomplete.

Here we consider a particular but physically important case in which $\gamma$ always vanishes at $r=0$ and thus $\dot{\gamma}$ should also always vanish there. Since $\dot{\gamma}=\left(\partial_{v} \gamma\right)_{u}+\left(\partial_{u} \gamma\right)_{v}=A-B$ by eq.(17), the condition $\left.\dot{\gamma}\right|_{r=0}=0$ leads

$$
A(t)=B(t)
$$

The above equation means that the same amount of outgoing null dust as that of ingoing one is emitted from the naked singularity as soon as the ingoing null dust hits the symmetry 
axis and forms the naked singularity there. In this case, the components of the metric tensor with respect to the Cartesian coordinate system are single valued and continuous because at the naked singularity $r=0, g_{t t}=-1=-g_{x x}=-g_{y y}=-g_{z z}$ and the other components vanishes. Thus even at the singularity, the norm $N$ of the radial geodesics is uniquely determined and further it is distinguishable whether the two vectors are parallel with each other. This fact guarantees that radial geodesics can be extended uniquely across the singularity. Non-radial causal geodesics might be unable to hit the singularity in the present case (see Appendix B), and therefore the present spacetime will be geodesically complete even though there is a spacetime singularity which satisfies LFC.

The completeness of radial geodesics means that the trajectories of the particles constituting the null dust are extendible and hence the condition (37) implies that each particle of the null dust passes through the naked singularity at the symmetry axis $r=0$. Therefore, the 'boundary condition' (37) at the naked singularity may be called passing-through condition. Further it should be noted that after all of the collapsed null dust leaves the naked singularity in the form of the outgoing one, the singularity disappears and the flat spacetime remains.

\section{SUMMARY AND DISCUSSION}

We studied the gravitational collapse of cylindrically symmetric null dust to form a naked singularity at its symmetry axis. We assumed on the situation in which the null dust is emitted from the naked singularity formed by the collapsed null dust. Then we investigated the curvature strength of the naked singularity and the behavior of geodesics near this naked singularity to see the back-reaction effect by the emission of null dust from the naked singularity.

We found that if the same amount of outgoing null dust as that of ingoing one is emitted from the naked singularity as soon as the ingoing null dust hits the symmetry axis and forms the naked singularity there, the spacetime will be geodesically complete, even if there is the naked singularity which satisfies LFC. The completeness of radial geodesics means that the trajectories of the particles constituting the null dust are uniquely extendible across the naked singularity and hence this case is regarded as the situation in which each particle of the null dust passes through the naked singularity and then leaves for infinite. Therefore, this 
boundary condition at the naked singularity has been named the passing-through condition. Further it should be noted that after all of the collapsed null dust leaves the naked singularity in the form of the outgoing null dust, the singularity disappears and the flat spacetime remains.

Usually, LFC is thought as a quite strong condition for a singularity [16]. However in the case of passing-through condition, we can extend geodesics across the singularity and resolve it. Thus, this singularity seems to be not so strong and severe. It implies that LFC does not necessarily represent the strength of spacetime singularities.

If all of the interactions becomes weaker in higher energy state due to the asymptotic freedom, the passing-through condition on the null dust seems to be natural. Thus null dust collapse with passing-through condition might be a toy model describing the essence of a "realistic naked singularity formation".

\section{Acknowledgments}

It is our pleasure to thank Hideki Ishihara for his valuable discussion and also to thank Hideo Kodama for his important comment on the geodesically completeness. We are grateful to colleagues in the astrophysics and gravity group of Osaka City University for helpful discussion and criticism. This work is supported by the Grant in Aid for Scientific Research (No.16540264) from JSPS. YK thanks the Yukawa Memorial Foundation for its support.

\section{APPENDIX A: RADIAL GEODESICS}

We derive the tangent vectors of radial geodesics in Morgan spacetime discussed in section

III. Here we adopt the null coordinate $u$ and $v$ and thus the Lagrangian of a geodesic is given by

$$
L=-e^{2 \gamma} \frac{d u}{d \lambda} \frac{d v}{d \lambda}+\left(\frac{d z}{d \lambda}\right)^{2}
$$


where $\lambda$ is the affine parameter. The geodesic equations for the radial geodesic are

$$
\begin{aligned}
\frac{d^{2} v}{d \lambda^{2}}+2\left(\partial_{v} \gamma\right)_{u}\left(\frac{d v}{d \lambda}\right)^{2} & =0 \\
\frac{d^{2} u}{d \lambda^{2}}+2\left(\partial_{u} \gamma\right)_{v}\left(\frac{d u}{d \lambda}\right)^{2} & =0 \\
\frac{d^{2} z}{d \lambda^{2}} & =0
\end{aligned}
$$

Substituting eq.(6) into the above equation, we obtain

$$
\frac{d}{d \lambda}\left(e^{2 \alpha} \frac{d v}{d \lambda}\right)=0, \quad \frac{d}{d \lambda}\left(e^{2 \beta} \frac{d u}{d \lambda}\right)=0 .
$$

Since the integrations of the above equations and eq.(A4) are trivial, we obtain

$$
e^{2 \alpha} \frac{d v}{d \lambda}=\text { const. }, \quad e^{2 \beta} \frac{d u}{d \lambda}=\text { const } \quad \text { and } \quad \frac{d z}{d \lambda}=\text { const. }
$$

Therefore for the null geodesics, we get

$$
\begin{aligned}
& \frac{d t}{d \lambda}=\frac{1}{2}\left[\left(\omega+k_{(\mathrm{r})}\right) e^{-2 \alpha(v)}+\left(\omega-k_{(\mathrm{r})}\right) e^{-2 \beta(u)}\right] \\
& \frac{d r}{d \lambda}=\frac{1}{2}\left[\left(\omega+k_{(\mathrm{r})}\right) e^{-2 \alpha(v)}-\left(\omega-k_{(\mathrm{r})}\right) e^{-2 \beta(u)}\right] \\
& \frac{d z}{d \lambda}=k_{(\mathrm{z})},
\end{aligned}
$$

where $\omega, k_{(\mathrm{r})}$ and $k_{(\mathrm{z})}$ are integration constants which satisfy $\omega^{2}=k_{(\mathrm{r})}^{2}+k_{(\mathrm{z})}^{2}$. Note that in the case of $\alpha=\beta=0$, the components of the tangent vector become

$$
\frac{d t}{d \lambda}=\omega, \quad \frac{d r}{d \lambda}=k_{(\mathrm{r})} \quad \text { and } \quad \frac{d z}{d \lambda}=k_{(\mathrm{z})}
$$

On the other hand, for the timelike geodesics, we obtain

$$
\begin{aligned}
& \frac{d t}{d \lambda}=\frac{\left(1+V_{(\mathrm{r})}\right) e^{-2 \alpha}+\left(1-V_{(\mathrm{r})}\right) e^{-2 \beta}}{2 \sqrt{1-V^{2}}}, \\
& \frac{d r}{d \lambda}=\frac{\left(1+V_{(\mathrm{r})}\right) e^{-2 \alpha}-\left(1-V_{(\mathrm{r})}\right) e^{-2 \beta}}{2 \sqrt{1-V^{2}}}, \\
& \frac{d z}{d \lambda}=\frac{V_{(\mathrm{z})}}{\sqrt{1-V^{2}}},
\end{aligned}
$$

where $V_{(\mathrm{r})}$ and $V_{(\mathrm{z})}$ are integration constants and $V^{2}=V_{(\mathrm{r})}^{2}+V_{(\mathrm{z})}^{2}$ should be smaller than unity. Note that in the case of $\alpha=\beta=0$, the components of the tangent vector become

$$
\frac{d t}{d \lambda}=\frac{1}{\sqrt{1-V^{2}}}, \quad \frac{d r}{d \lambda}=\frac{V_{(\mathrm{r})}}{\sqrt{1-V^{2}}} \quad \text { and } \quad \frac{d z}{d \lambda}=\frac{V_{(\mathrm{z})}}{\sqrt{1-V^{2}}}
$$




\section{APPENDIX B: NON-RADIAL GEODESICS NEAR THE SINGULARITY}

We investigate the behavior of non-radial geodesics near the naked singularity in the case of $A(t)=B(t)$. Adopting the coordinate system (1), the geodesic equations are written as

$$
\begin{aligned}
\frac{d}{d \lambda}\left(e^{2 \gamma} \frac{d t}{d \lambda}\right) & =-\dot{\gamma} e^{2 \gamma}\left[-\left(\frac{d t}{d \lambda}\right)^{2}+\left(\frac{d r}{d \lambda}\right)^{2}\right] \\
\frac{d}{d \lambda}\left(e^{2 \gamma} \frac{d r}{d \lambda}\right) & =\gamma^{\prime} e^{2 \gamma}\left[-\left(\frac{d t}{d \lambda}\right)^{2}+\left(\frac{d r}{d \lambda}\right)^{2}\right]+r\left(\frac{d \phi}{d \lambda}\right)^{2}, \\
\frac{d}{d \lambda}\left(r^{2} \frac{d \phi}{d \lambda}\right) & =0 \\
\frac{d^{2} z}{d \lambda^{2}} & =0
\end{aligned}
$$

where $\lambda$ is the affine parameter. The third and forth equations can be easily integrated once and we obtain

$$
r^{2} \frac{d \phi}{d \lambda}=L, \quad \frac{d z}{d \lambda}=P
$$

where $L$ and $P$ are integration constants. The non-radial geodesic means the geodesic with non-vanishing $L$. Then the normalization of the geodesic tangent vector leads

$$
\left(\frac{d r}{d \lambda}\right)^{2}=\left(\frac{d t}{d \lambda}\right)^{2}+e^{-2 \gamma}\left(\chi-\frac{L^{2}}{r^{2}}-P^{2}\right),
$$

where $\chi$ is equal to -1 for timelike geodesics, unity for spacelike geodesics and vanishes for null geodesics. Since the left hand side of the above equation is non-negative, we obtain an inequality as

$$
r^{2} \geq \frac{L^{2}}{e^{2 \gamma}(d t / d \lambda)^{2}-\left(P^{2}-\chi\right)} .
$$

Hence in order that the geodesic of non-vanishing $L$ hits the naked singularity at $r=0$, $d t / d \lambda$ should become infinite in approaching to the naked singularity.

Here we adjust the affine parameter $\lambda$ and coordinate time $t$ so that the geodesic hits the singularity at $\lambda=0$ and $t=0$. Thus, the geodesic which hits the naked singularity should behave near the naked singularity as

$$
t \sim C_{(\mathrm{t})} \lambda^{p} \quad \text { and } \quad r \sim C_{(\mathrm{r})} \lambda^{q}
$$

where $C_{(\mathrm{t})}$ and $C_{(\mathrm{r})}$ are constant, and $0<p<1$ should be satisfied since $d t / d \lambda \rightarrow \infty$ for $\lambda \rightarrow \infty$. 
Hereafter our purpose is to show that there is no solution consistent with the asymptotic

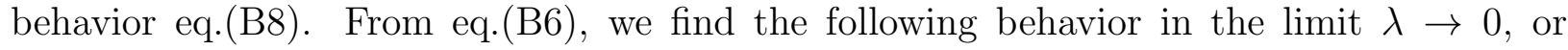
equivalently, $r \rightarrow 0$,

$$
\left(\frac{d r}{d \lambda}\right)^{2} \sim\left(\frac{d t}{d \lambda}\right)^{2}-e^{-2 \gamma_{0}} \frac{L^{2}}{r^{2}}
$$

where $\gamma_{0}$ is the value of $\gamma$ when the geodesic hits the naked singularity. Of course, the following inequality should be satisfied:

$$
\left(\frac{d t}{d \lambda}\right)^{2}>e^{-2 \gamma_{0}} \frac{L^{2}}{r^{2}} \text { for } \lambda \longrightarrow 0 .
$$

Therefore $q$ should be equal to $p$ and further from the above inequality, we obtain $p \leq 1 / 2$, because the left hand side is proportional to $\lambda^{2(p-1)}$ while the right hand side is proportional to $\lambda^{-2 q}=\lambda^{-2 p}$.

By using the normalization condition of the tangent vector, eq.(B1) is rewritten as

$$
\frac{d^{2} t}{d \lambda^{2}}+2 \dot{\gamma}\left(\frac{d t}{d \lambda}\right)^{2}+2 \gamma^{\prime}\left(\frac{d t}{d \lambda}\right)\left(\frac{d r}{d \lambda}\right)-\dot{\gamma} e^{-2 \gamma}\left(P^{2}+\frac{L^{2}}{r^{2}}-\chi\right)=0
$$

We consider the case in which $\gamma^{\prime}, \ddot{\gamma}$ and $\dot{\gamma}^{\prime}$ are finite even at the naked singularity $r=0$ and their values at the naked singularity are denoted by $\gamma_{0}^{\prime}, \ddot{\gamma}_{0}$ and $\dot{\gamma}_{0}^{\prime}$, respectively. Note that $\dot{\gamma}$ vanishes at $r=0$ by the assumption $A(t)=B(t)$ and thus it behaves near the naked singularity as

$$
\dot{\gamma} \sim\left(\ddot{\gamma}_{0} \frac{d t}{d \lambda}+\dot{\gamma}_{0}^{\prime} \frac{d r}{d \lambda}\right) \lambda \sim\left(\ddot{\gamma}_{0} C_{(\mathrm{t})}+\dot{\gamma}_{0}^{\prime} C_{(\mathrm{r})}\right) p \lambda^{p} .
$$

Keep the above behavior in mind and substituting the asymptotic behavior eq.(B8) into eq.(B11), we obtain

[The left hand side of eq.(B11)]

$$
\begin{aligned}
& \sim p(p-1) C_{(\mathrm{t})} \lambda^{p-2}+2\left(\ddot{\gamma}_{0} C_{(\mathrm{t})}+\dot{\gamma}_{0}^{\prime} C_{(\mathrm{r})}\right) C_{(\mathrm{t})} p^{2} \lambda^{3 p-2}+2 \gamma_{0}^{\prime} C_{(\mathrm{t})} C_{(\mathrm{r})} p^{2} \lambda^{2(p-1)} \\
& \quad-\left(\ddot{\gamma}_{0} C_{(\mathrm{t})}+\dot{\gamma}_{0}^{\prime} C_{(\mathrm{r})}\right) p e^{-2 \gamma_{0}} \frac{L^{2}}{C_{(\mathrm{r})}^{2}} \lambda^{-p} .
\end{aligned}
$$

The leading order terms should be balanced with each other. One can easily see that the second term can not be one of the leading order terms. If the 2 nd term is so, $3 p-2$ should be less than or equal to each of $p-2,2(p-1)$ and $(-p)$, because we consider the limit $\lambda \rightarrow 0$. This leads $p \leq 0$, conflicting with $p>0$. Thus this is impossible. If the 1 st term is balanced with the 3 rd term, then $p-2=2(p-1)$. This leads $p=0$ and thus it is impossible because $p>0$. If the 1 st term is balanced with the last term, then $p-2=-p$ and this 
leads $p=1$. This conflicts with $p \leq 1 / 2$ and thus this is impossible. Finally, if the 3rd term is balanced with the last term, $2(p-1)=-p$ and this leads $p=2 / 3$. This also conflicts with $p \leq 1 / 2$. Therefore there is no solution which behaves as eq. (B8). This fact strongly suggests that there is no non-radial geodesic which hits the naked singularity at $r=0$.

\section{APPENDIX C: WEYL TENSOR}

We investigate the behavior of Weyl tensor in approaching to the neked singularity. The components of Weyl tensor in the coordinate system (11) are

$$
\begin{aligned}
& C_{t \phi t \phi}=C_{r \phi r \phi}=-r^{2} C_{t z t z}=-r^{2} C_{r z r z}=\frac{1}{2} r \gamma^{\prime} \\
& C_{t \phi t \phi}=-r^{2} C_{t z r z}=\frac{1}{2} r \dot{\gamma},
\end{aligned}
$$

and the other components vanish, where $\dot{\gamma}=\left(\partial_{t} \gamma\right)_{r}$ and $\gamma^{\prime}=\left(\partial_{r} \gamma\right)_{t}$. In order to investigate their behavior along the null geodesic defined by (A7), (A8) and (A9), we intorduce a parallely propagating frame as follows:

$$
\begin{aligned}
e_{(\lambda)}^{\mu} & =\left(\frac{\left(\omega+k_{(r)}\right) e^{-2 \alpha}+\left(\omega-k_{(r)}\right) e^{-2 \beta}}{2}, \frac{\left(\omega+k_{(r)}\right) e^{-2 \alpha}-\left(\omega-k_{(r)}\right) e^{-2 \beta}}{2}, 0, k_{(z)}\right)_{t r \phi z} \\
e_{(\ell)}^{\mu} & =\left(\frac{e^{-2 \alpha}+e^{-2 \beta}}{2\left(\omega-k_{(z)}\right)}, \frac{e^{-2 \alpha}-e^{-2 \beta}}{2\left(\omega-k_{(z)}\right)}, 0, \frac{1}{\omega-k_{(z)}}\right)_{t r \phi z} \\
e_{(\phi)}^{\mu} & =\left(0,0, \frac{1}{r}, 0\right)_{t r \phi z}, \\
e_{(s)}^{\mu} & =\left(\frac{c_{\alpha} e^{-2 \alpha}-c_{\beta} e^{-2 \beta}}{2\left(\omega-k_{(z)}\right)}, \frac{c_{\alpha} e^{-2 \alpha}+c_{\beta} e^{-2 \beta}}{2\left(\omega-k_{(z)}\right)}, 0, \frac{k_{(r)}}{\omega-k_{(z)}}\right)_{t r \phi z}
\end{aligned}
$$

where we set $c_{\alpha}=\omega+k_{(r)}-k_{(z)}, c_{\beta}=\omega-k_{(r)}-k_{(z)}$. These vectors satisfy the following relations:

$$
\begin{aligned}
& e_{(a)}^{\mu} e_{(a) \mu}=0, \quad e_{(\lambda)}^{\mu} e_{(\ell) \mu}=-1, \quad(a=\lambda, \ell), \\
& e_{(b)}^{\mu} e_{(b) \mu}=1, \quad e_{(s)}^{\mu} e_{(\phi) \mu}=0, \quad e_{(a)}^{\mu} e_{(b) \mu}=0, \quad(b=s, \phi) \\
& e_{(a) \mu ; \nu} e_{(\lambda)}^{\nu}=e_{(b) \mu ; \nu} e_{(\lambda)}^{\nu}=0 .
\end{aligned}
$$

In this frame, the non-vanishing components of the Weyl tensor in the form of $C_{(\lambda)(*)(\lambda)(*)}$ are

$$
\begin{aligned}
C_{(\lambda)(\phi)(\lambda)(\phi)} & =\frac{\alpha_{, v} e^{-4 \alpha}\left(\omega+k_{(r)}\right)^{2}-\beta_{, u} e^{-4 \beta}\left(\omega-k_{(r)}\right)^{2}}{2 r} \\
C_{(\lambda)(s)(\lambda)(s)} & =\frac{-\alpha_{, v} e^{-4 \alpha}\left(\omega+k_{(r)}\right)^{2}+\beta_{, u} e^{-4 \beta}\left(\omega-k_{(r)}\right)^{2}}{2 r} .
\end{aligned}
$$


Thus, all the above components have the following behavior:

$$
C_{(\lambda)(*)(\lambda)(*)}=\frac{f(\lambda)}{r}
$$

where $f(\lambda)$ is a function having non-zero finite value at $\lambda=0$. We set the following limiting value as $Y$ :

$$
\begin{aligned}
Y & :=\lim _{\lambda \rightarrow 0} \frac{\int_{\lambda_{0}}^{\lambda} d \lambda^{\prime}\left|f\left(\lambda^{\prime}\right) / r\right|}{\log \lambda}=\lim _{\lambda \rightarrow 0} \frac{\lambda}{r}|f(\lambda)|=\lim _{\lambda \rightarrow 0} \frac{d \lambda}{d r}|f(0)| \\
& =\frac{2|f(0)|}{\left(\omega+k_{r}\right) e^{-2 \alpha}-\left.\left(\omega-k_{r}\right) e^{-2 \beta}\right|_{\lambda=0}},
\end{aligned}
$$

where $\lambda_{0}$ is some negative value. In order to show the convergence of the limit (19), let us consider the following limit:

$$
\begin{aligned}
\lim _{\lambda \rightarrow 0} \frac{\int_{\lambda_{0}}^{\lambda} d \lambda^{\prime} \int_{\lambda_{0}}^{\lambda^{\prime}} d \lambda^{\prime \prime}\left(\int_{\lambda_{0}}^{\lambda^{\prime \prime}} d \lambda^{\prime \prime \prime}\left|f\left(\lambda^{\prime \prime \prime}\right) / r\right|\right)^{2}}{\frac{1}{2} \lambda^{2}(\log \lambda)^{2}-\frac{3}{2} \lambda^{2} \log \lambda+\frac{7}{4} \lambda^{2}} & =\lim _{\lambda \rightarrow 0} \frac{\int_{\lambda_{0}}^{\lambda} d \lambda^{\prime}\left(\int_{\lambda_{0}}^{\lambda^{\prime}} d \lambda^{\prime \prime}\left|f\left(\lambda^{\prime \prime}\right) / r\right|\right)^{2}}{\lambda(\log \lambda)^{2}-2 \lambda \log \lambda+2 \lambda} \\
& =\lim _{\lambda \rightarrow 0} \frac{\left(\int_{\lambda_{0}}^{\lambda} d \lambda^{\prime}\left|f\left(\lambda^{\prime}\right) / r\right|\right)^{2}}{(\log \lambda)^{2}}=Y^{2}
\end{aligned}
$$

where we have used the l'Hospital theorem. Therefore, we have

$$
\begin{aligned}
\lim _{\lambda \rightarrow 0} \int_{\lambda_{0}}^{\lambda} d \lambda^{\prime} \int_{\lambda_{0}}^{\lambda^{\prime}} d \lambda^{\prime \prime} & \left(\int_{\lambda_{0}}^{\lambda^{\prime \prime}} d \lambda^{\prime \prime \prime}|f(\lambda) / r|\right)^{2} \\
& =Y^{2} \lim _{\lambda \rightarrow 0}\left(\frac{1}{2} \lambda^{2}(\log \lambda)^{2}-\frac{3}{2} \lambda^{2} \log \lambda+\frac{7}{4} \lambda^{2}\right) \\
= & 0 .
\end{aligned}
$$

Thus, for all non-vanishing Weyl components, the limit (19) converges.

\section{APPENDIX D: SCST FOR TIMELIKE GEODESICS}

In this Appedix, we show that the naked singularity does not satisfy the necessary condition for SCST in the case of timelike geodesics. The necessary condition in this case is as follows: for some component $R_{(\tau)(i)(\tau)(j)}$ of the Riemann tensor in a parallely propagated frame, the integral

$$
I_{(i)(j)}(\tau)=\int_{\tau_{0}}^{\tau} d \tau^{\prime} \int_{\tau_{0}}^{\tau^{\prime}} d \tau^{\prime \prime}\left|R_{(\tau)(i)(\tau)(j)}\left(\tau^{\prime \prime}\right)\right|
$$


does not converge as $\tau \rightarrow 0$, where the proper time $\tau$ is adjusted as it equals zero at $r=0$ and $\tau_{0}$ is some negative value. A parallely propagating frame along the timelike geodesic (8) $-(10)$ is

$$
\begin{aligned}
& e_{(\tau)}^{\mu}=\left(\frac{d t}{d \tau}, \frac{d r}{d \tau}, 0, \frac{d z}{d \tau}\right)_{t r \phi z}, \\
& e_{(n)}^{\mu}=\frac{1}{\sqrt{1+(d z / d \tau)^{2}}}\left(\frac{d r}{d \tau}, \frac{d t}{d \tau}, 0,0\right)_{t r \phi z}, \\
& e_{(\phi)}^{\mu}=\left(0,0, \frac{1}{r}, 0\right)_{t r \phi z}, \\
& e_{(u)}^{\mu}=\frac{1}{\sqrt{1+(d z / d \tau)^{2}}}\left(\frac{d z}{d \tau} \frac{d r}{d \tau}, \frac{d z}{d \tau} \frac{d t}{d \tau}, 0,1+\left(\frac{d z}{d \tau}\right)^{2}\right)_{t r \phi z} .
\end{aligned}
$$

The only non-vanishing component of the Rimann tensor in this frame is

$$
R_{(\tau)(\phi)(\tau)(\phi)}=\frac{1}{r\left(1-V^{2}\right)}\left[\left(1+V_{(r)}\right)^{2} A e^{-4 \alpha}+\left(1-V_{(r)}\right)^{2} B e^{-4 \beta}\right] .
$$

We set the following limiting value as $Z$ :

$$
\begin{aligned}
Z: & =\lim _{\tau \rightarrow 0} \tau\left|R_{(\tau)(\phi)(\tau)(\phi)}\right| \\
& =\lim _{\tau \rightarrow 0} \frac{\tau}{r}\left|\frac{1}{\left(1-V^{2}\right)}\left[\left(1+V_{(r)}\right)^{2} A e^{-4 \alpha}+\left(1-V_{(r)}\right)^{2} B e^{-4 \beta}\right]\right| \\
& =\lim _{\tau \rightarrow 0} \frac{d \tau}{d r}\left|\frac{1}{\left(1-V^{2}\right)}\left[\left(1+V_{(r)}\right)^{2} A e^{-4 \alpha}+\left(1-V_{(r)}\right)^{2} B e^{-4 \beta}\right]\right| \\
& =\frac{2}{\sqrt{1-V^{2}}}\left|\frac{\left(1+V_{(r)}\right)^{2} A e^{-4 \alpha}+\left(1-V_{(r)}\right)^{2} B e^{-4 \beta}}{\left(1+V_{(r)}\right) e^{-2 \alpha}+\left(1-V_{(r)}\right) e^{-2 \beta}}\right|_{\tau=0},
\end{aligned}
$$

where we have used the l'Hopital theorem. Then,

$$
\begin{aligned}
\lim _{\tau \rightarrow 0} \frac{\int_{\tau_{0}}^{\tau} d \tau^{\prime} \int_{\tau_{0}}^{\tau^{\prime}} d \tau^{\prime \prime}\left|R_{(\tau)(\phi)(\tau)(\phi)}\left(\tau^{\prime \prime}\right)\right|}{\tau \ln \tau-\tau} & =\lim _{\tau \rightarrow 0} \frac{\int_{\tau_{0}}^{\tau} d \tau^{\prime}\left|R_{(\tau)(\phi)(\tau)(\phi)}\left(\tau^{\prime}\right)\right|}{\ln \tau} \\
& =\lim _{\tau \rightarrow 0} \tau\left|R_{(\tau)(\phi)(\tau)(\phi)}(\tau)\right|=Z .
\end{aligned}
$$

Thereofre, we have

$$
I_{(i)(j)}(\tau)=\int_{\tau_{0}}^{\tau} d \tau^{\prime} \int_{\tau_{0}}^{\tau^{\prime}} d \tau^{\prime \prime}\left|R_{(\tau)(i)(\tau)(j)}\left(\tau^{\prime \prime}\right)\right|=Z \lim _{\tau \rightarrow 0}(\tau \ln \tau-\tau)=0 .
$$

The singularity does not satisfy the necessary condition for SCST.

[1] R. Penrose, Riv. Nuovo Cimento 1, 252 (1969). 
[2] P. Joshi, Global Aspects in Gravitation and Cosmology (Oxford University Press, New York, 1993).

[3] T. Nakamura, K. Maeda, S. Miyama, M. Sasaki, in Proceedings of the 2nd Marcel Grossmann Meeting on General Relativity edited by R. Ruffini, (North-Holland, Amsterdam, 1982), p. 675.

[4] T. Nakamura and H. Sato, Prog. Theor. Phys. 67, 1396 (1982).

[5] S. L. Shapiro and S. A. Teukolsky, Phys. Rev. Lett. 66, 994 (1991).

[6] T. Harada and K. Nakao, Phys. Rev. D 70, 041501 (2004).

[7] K. S. Thorne, in Magic Without Magic; John Archibald Wheeler, edited by J. Klauder, (Frieman, San Francisco, 1972), p. 231.

[8] S. A. Hayward, Class. Quant. Grav. 17, 1749 (2000).

[9] T. A. Morgan, Gen. Rel. Grav. 4, 273 (1973), p. 273.

[10] P. S. Letelier and A. Wang, Phys. Rev. D 49, 5105 (1994).

[11] S. W. Hawking and G. F. R. Ellis, The large scale structure of space-time, (Cambridge university press, Cambridge, 1973).

[12] M. A. Melvin, Phys. Lett. 8, 65 (1964).

[13] M. A. Melvin, Phys. Rev. 139, B225 (1965).

[14] B. C. Nolan, Phys. Rev. D 65, 104006 (2002).

[15] A. Królak, J. Math. Phys. 28, 138 (1987).

[16] C. J. S. Clarke, The Analysis of Space-Time Singularities, (Cambridge University Press, Cambridge, 1993) Chapter 8. 